\title{
A web-based application for estimating energy requirements in elderly patients
}

\section{Abstract}

Elderly patients are at risk of malnutrition and need an appropriate assessment of energy requirements. In the clinical setting, predictive equations are widely used to estimate the Resting Energy Expenditure (REE). Although easy to use, these equations are not always validated in the elderly and, even if validated, they often provide different outputs of energy requirements for the same subject. The aim of the present work is to develop a web-based application helping clinicians in finding out the most appropriate equation for estimating the REE for each subject. The web-based application is based on a systematic review of the equations for the estimation of REE in the elderly. The systematic review was carried out on PubMed and Scopus following the PRISMA guidelines. Studies in subjects older than 65 years of age that tested the performance of a predictive equation for the estimation of REE vs. a gold standard (indirect calorimetry or doubly labeled water) were included in the review. Studies performed in critically ill elderly patients were excluded. The initial search identified 2035 studies. The final review included 50 studies. Included studies were mainly observational, conducted in healthy elderly subjects enrolled in the outpatient setting, and using indirect calorimetry as the gold standard. The 50 studies included in the review corresponded to 189 different equations. Several parameters were included in the equations, and they can be divided as following: anthropometric characteristics, body composition parameters, environmental measures, laboratory tests, the presence of comorbidities, and physical activity frequency. The equations retrieved were tested on a sample of 88 subjects aged $>65$ years enrolled in an Italian nursing home. Based on the systematic review and the pilot testing of the equations, it has been developed a web application (http://r-ubesp.dctv.unipd.it:3838/equationer) that allows for the estimation of REE using the equation most appropriate according to the subject's characteristic and parameters available. The assessment of the energy requirements in the elderly is crucial for the management of nutritional problems in this population group since nutritional problems are related to worse health outcomes. The present study showed a wide use of different type of equations for the estimation of REE in the elderly highlighting the need of choosing the most appropriate predictive equation according to the subject characteristics and health status. The web application will help clinicians in doing that.

\section{Conflict of Interest}

There is no conflict of interest 